\section{P18.14 SEX WORKER OUTREACH SCREENING IN WESTERN SYDNEY; EARLY IDENTIFICATION OF A HIGH- RISK POPULATION}

R Varma, M Ma, J Walsh, M Power*, A Rochford. Western Sydney Sexual Health Centre, Parramatta, Sydney

\subsection{6/sextrans-2015-052270.637}

Introduction The Western Sydney Local Health District (WSLHD) serves a culturally and linguistically diverse (CALD) population. Sex workers (SW) from this community may not access health care facilities or be aware of sexually transmitted infection (STI) prevention strategies. We describe a tailored outreach screening of female SW in WSLHD.

Methods A trained health care interpreter (Mandarin) assisted in face- to- face consultations and self collected written sexual history; registered nurses provided weekly screening for STIs and blood borne viruses (BBV) in brothels from July 2014-March 2015.

Results 133 SW, comprising 10 different nationalities, at 28 different commercial venues were screened. 38/133 (28.6\%) were new clients to our service. 65/133 (48.9\%) were born in China, followed by $33 / 133$ (22.5\%) Thai and 17/133 (12.8\%) Australasian. Mean age 41.6, 29.4 and 32 years for Chinese, Thai and Australasian born SW respectively. 30/133 (22.4\%) had been in Australia for less than 12 months, 45/133 (31.6\%) unknown duration. 19/133 (14.3\%) reported being a SW for less than 6 months, $42 / 133(31.3 \%)$ for 12 months or less, 35/133 (26.3\%) unknown.

20/133 (15\%) and 50/133 (37.6\%) of SW reported less than $100 \%$ condom use for vaginal sex and oral sex with clients respectively. 31/50 (62\%) of SW reporting inconsistent condom use for oral sex were from China.

14/133 (10.5\%) denied previous HIV screening. 21\% of SW were diagnosed with an STI including: gonorrhoea 12/133 (9\%, four SW had multi-site infection), chlamydia 14/133 (10.4\%, four SW had multi- site infections), chronic hepatitis B (5\%) and $2 \%$ syphilis. No HIV diagnoses were made.

Conclusion This outreach initiative has identified a significant population of potentially vulnerable SW from SE Asian countries with high rates of unsafe sexual practice and STIs. This predominantly Mandarin speaking population may not access existing health promotion and screening services; a targeted, ongoing outreach is warranted.

Disclosure of interest statement No disclosures.

\section{P18.15 A POCT- ADAPTABLE TEST FOR THE SIMULTANEOUS IDENTIFICATION OF N. GONORRHOEAE AND ITS CIPROFLOXACIN SUSCEPTIBILITY STATUS}

${ }^{1} \mathrm{JR}$ Dillon*, ${ }^{1} \mathrm{~A}$ Taheri, ${ }^{1} \mathrm{NH}$ Khan, ${ }^{1} \mathrm{RP}$ Parti, ${ }^{2} \mathrm{~A}$ Kusalik. ${ }^{1}$ Vaccine and Infectious Diseases Organization, Saskatoon, SK, Canada; ${ }^{2}$ Department of Computer Science, University of Saskatchewan, Saskatoon, Canada

$10.1136 /$ sextrans-2015-052270.638

Introduction Every year, Neisseria gonorrhoeae $(\mathrm{Ng})$ causes 106 million new gonorrhoea infections worldwide and has recently joined the fast-expanding group of multi-drug resistant superbugs. Treatment is often empirical/delayed as culture-based antibiotic susceptibility tests in current use can take several days. A Point-of-Care Test (POCT) for antimicrobial susceptibility would change this; for example, no longer recommended antibiotics such as ciprofloxacin could be used in certain regions where high percentages of isolates remain susceptible. The objective of the present study was to develop and validate a portfolio of diagnostic primer pairs for application in a Nucleic Acid Amplification-based POCT for the concurrent diagnosis of $\mathrm{Ng}$ and ciprofloxacin susceptibility.

Methods A bioinformatics analysis of $>30,000$ bacterial genomes identified 9 unique signature sequences specific to $\mathrm{Ng}$ FA1090 that were used as targets in a SYBR green-based qPCR assay. 9 diagnostic primer pairs were evaluated for specificity and sensitivity on $271 \mathrm{Ng}$ and non-gonococcal isolates. Two primer pairs targeting ciprofloxacin resistance-conferring single nucleotide polymorphisms in gyrA were tested on 200 resistant and susceptible $\mathrm{Ng}$ isolates. qPCR was performed by an Applied Biosystems StepOnePLusTM qPCR system.

Results Out of the 9 diagnostic primer pairs tested for Ng identification, one probe failed to detect 28 positive samples of $\mathrm{Ng}$ out of a total of 234 isolates. Another primer pair, which amplifies the DR9 repeat region used in the COBAS4800 was not able to detect one $\mathrm{Ng}$ isolate collected from Hong Kong. The remaining 7 primer pairs showed 100\% specificity in terms of $\mathrm{Ng}$ detection and were highly sensitive in detecting $\mathrm{Ng}$ DNA in concentrations as low as $0.00001 \mathrm{ng} / \mathrm{ul}$. A multiplexed assay using ciprofloxacin susceptibility-determining primer pairs distinctly differentiated between resistant and susceptible isolates based on melt curve analysis.

Conclusion A POCT-adaptable assay has been developed for the simultaneous identification of $N$. gonorrhoeae and its ciprofloxacin susceptibility status.

Disclosure of interest statement The present work was supported by Grand Challenges Canada ((\#S5 398). No grants were received from any company in the development of this study. 